\title{
Manual of Pediatric Anesthesia - Seventh Edition
}

\author{
Jerrold Lerman, Charles J. Cote, David J. Steward (Editors). Springer International \\ Publishing AG Switzerland, 2016. ISBN 978-3-319-30682-7
}

\author{
David Faraoni, MD, PhD, FCCP
}

Received: 30 January 2017/Revised: 20 March 2017/Accepted: 12 April 2017/Published online: 18 April 2017

(C) Canadian Anesthesiologists' Society 2017

The Manual of Pediatric Anesthesia (Seventh Edition) is a concise handbook that summarizes useful information for anesthesiologists caring for infants and children. The previous version was published in 2010 by the same three authors, and this edition is consistent in terms of presentation and content. Its larger size is noteworthy, although it arguably decreases the portability of a product considered, in the title, as a manual. This handbook describes management strategies for various anesthesia subspecialties and a range of options for addressing challenging clinical situations. Although it contains an impressive amount of information, its relatively portable format makes it a convenient reference source that can be brought into the operating room. Throughout the book, readers are provided with informative tables and algorithms summarizing key points that could help guide preparation of a perioperative anesthesia plan.

The book includes 18 chapters and 3 appendices. Chapter 1 focuses on the foundations of pediatric anesthesia, and Chapter 2 reviews basic anatomy and physiology. Chapter 3 summarizes the pharmacological properties and doses of the drugs commonly used in pediatric anesthesia, including inhalation agents and intravenous hypnotics, opioids, and neuromuscular blocking drugs. It also briefly describes drugs used for postoperative pain management. Chapter 4 summarizes common techniques and procedures used in pediatric anesthesia, providing basic information on premedication, airway management (including the difficult airway),

D. Faraoni, MD, PhD, FCCP ( $₫)$

Department of Anesthesia and Pain Medicine, The Hospital for

Sick Children, University of Toronto, Toronto, ON, Canada

e-mail: david.faraoni@mail.utoronto.ca ventilation, and monitoring. The chapter also addresses important topics such as the management of body temperature and fluid balance, as well as blood product transfusion. Chapter 5 is a brief review of locoregional techniques, offering only limited information. The next edition would benefit from the addition of figures and images representing landmarks for each of the techniques described. Chapter 6 reviews a variety of medical conditions that may influence anesthetic management. Although not exhaustive, it reviews the most prevalent co-morbidities found in pediatric patients and includes a list of relevant complementary articles that could be helpful. Chapter 6 is also linked to Appendix A, which summarizes anesthesia challenges in the presence of syndromes and unusual disorders. The information contained therein is easily accessible, making it a helpful, first-line resource with references to complementary, more detailed information. Chapter 7 reviews postoperative care and pain management. There is considerable overlap with Chapter 3 concerning pain management drugs, however, which perhaps could be addressed in the manual's next version. Another shortcoming of Chapter 7 is the too-brief discussion of critical postoperative events, including the criteria for safe patient discharge from the post-anesthesia care unit.

The other 11 chapters discuss anesthesia management for all subspecialties, including neurosurgery and invasive neuroradiology, ophthalmology, otorhinolaryngology, dental surgery, plastic and reconstructive surgery, thoracoabdominal surgery, cardiovascular surgery and cardiology procedures, orthopedic surgery, urology, trauma, and acute burns. It also addresses anesthesia provision outside the operating room. Although not exhaustive, these chapters contain the relevant 
information an anesthesiologist would require to provide informed anesthesia care to a pediatric patient.

The last 117 pages of the handbook comprise three appendices. The first appendix (A) is a detailed table summarizing the implication of syndromes and unusual disorders for anesthesia. References for interesting articles addressing each syndrome and disorder are also provided. The last two appendices are extremely important as they review cardiopulmonary resuscitation (Appendix B) and drug doses (Appendix C).

In conclusion, the Manual of Pediatric Anesthesia is a concise, comprehensive handbook that summarizes useful information for anesthesiologists providing anesthesia care to infants and children. The provision of an index of pediatric syndromes is noteworthy and distinguishes it from other books of similar format. Although this manual cannot replace comprehensive textbooks concerned with pediatric anesthesia, it is a highly useful, portable, complementary text.

Conflicts of interest None declared.

Editorial responsibility This submission was handled by Dr. Steven Backman, Associate Editor, Canadian Journal of Anesthesia. 